\title{
On an Almost $C(\alpha)$-Manifold Satisfying Certain Conditions on the Concircular Curvature Tensor
}

\author{
Mehmet Atçeken, Umit Yildirim \\ Gaziosmanpasa University, Faculty of Arts and Sciences, Department of Mathematics, Tokat, Turkey \\ Email address: \\ mehmet.atceken382@gmail.com (M. Atçeken), umit.yildirim@gop.edu.tr (U. Yildirim)
}

\section{To cite this article:}

Mehmet Atçeken, Umit Yildirim. On an Almost $C(\alpha)$-Manifold Satisfying Certain Conditions on the Concircular Curvature Tensor. Pure and Applied Mathematics Journal. Special Issue: Applications of Geometry. Vol. 4, No. 1-2, 2015, pp. 31-34. doi: 10.11648/j.pamj.s.2015040102.18

\begin{abstract}
We classify almost $C(\alpha)$-manifolds, which satisfy the curvature conditions $\widetilde{Z}(\xi, X) R=0, \widetilde{Z}(\xi, X) \widetilde{Z}=0$, $\widetilde{Z}(\xi, X) S=0$ and $\widetilde{Z}(\xi, X) P=0$, where $\widetilde{Z}$ is the concircular curvature tensor, $P$ is the Weyl projective curvature tensor, $S$ is the Ricci tensor and $R$ is Riemannian curvature tensor of manifold.
\end{abstract}

Keywords: Almost $C(\alpha)$-Manifold, Concircular Curvature Tensor, Projective Curvature Tensor

\section{Introduction}

An odd-dimensional Riemannian manifold $(M, g)$ is said to be an almost co-Hermitian or almost contact metric manifold if there exist on $M$ a $(1,1)$ tensor field $\phi$, a vector field $\xi$ (called the structure vector field) and 1-form $\eta$ such that

$$
\begin{gathered}
\eta(\xi)=1, \quad \phi^{2}(X)=-X+\eta(X) \xi \\
g(\phi X, \phi Y)=g(X, Y)-\eta(X) \eta(Y) \\
\phi(\xi)=0, \quad \eta \circ \phi=0
\end{gathered}
$$

for any vector fields $X, Y$ on $M$.

The Sasaki form (or fundamental 2-form) $\Phi$ of an almost co-Hermitian manifold $(M, g, \phi, \xi, \eta)$ is defined by

$$
\Phi(X, Y)=g(X, \phi Y)
$$

for all $X, Y$ on $M$ and this form satisfies $\eta \Lambda \Phi^{n} \neq 0$. This means that every almost co-Hermitian manifold is orientable and $(\eta, \Phi)$ defines an almost cosymplectic structure on $M$. If this associated structure is cosymplectic $d \Phi=d \eta=0$, then $M$ is called an almost co-Kähler manifold. On the other hand, when $\Phi=d \eta$, the associated almost cosymplectic structure is a contact structure and is an almost Sasakian manifold. It is well known every contact manifold has an almost Sasakian structure.

The Nijenhuis tensor of type $(1,1)$-tensor field $\phi$ is type $(1,2)[\phi, \phi]$ defined by

$$
[\phi, \phi](X, Y)=\phi^{2}[X, Y]+[\phi X, \phi Y]-\phi[\phi X, Y]
$$

$-\phi[X, \phi Y]$

where $[X, Y]$ is the Lie bracket of $X, Y \in \chi(M)$.

On the other hand, an almost co-complex structure is called integrable if $[\phi, \phi]=0$ and normal $[\phi, \phi]+$ $2 d \eta \otimes \xi=0$. An integrable almost cocomplex structure is a cocomplex structure. A co-Kähler manifold (or normal cosymplectic manifold) is an integrable (or equivalently, a normal) almost co-Kähler manifold, while a Sasakian manifold is a normal almost contact metric manifold [3].

\section{Preliminaries}

In [2], $\mathrm{N}(\mathrm{k})-$ contact metric manifolds satisfying $\widetilde{\mathrm{Z}}(\xi, \mathrm{X}) \widetilde{\mathrm{Z}}=\widetilde{\mathrm{Z}}(\xi, \mathrm{X}) \mathrm{R}=\mathrm{R}(\xi, \mathrm{X}) \widetilde{\mathrm{Z}}=0$ were classified.

In [1], $\widetilde{Z}(\xi, X) R=R(\xi, X) R=\widetilde{Z}(\xi, X) S=\widetilde{Z}(\xi, X) C=0$ on $\mathrm{P}$-Sasakian manifolds and obtained the some results.

M. M. Tripathi and J. S. Kim gave a classification of $(\mu, \kappa)$-manifolds satisfying the conditions $\widetilde{\mathrm{Z}}(\xi, \mathrm{X}) \mathrm{S}=0$ [7].

Definition 2.1. An almost $C(\alpha)$-manifold $M$ is an almost co-Hermitian manifold such that the Riemann curvature tensor satisfies the following property, $\exists \alpha \in R$ such that $R(X, Y, W, Z)=R(X, Y, \phi Z, \phi W)$

$$
\begin{aligned}
& +\alpha\{-g(X, Z) g(Y, W)+g(X, W) g(Y, Z) \\
& +g(X, \phi Z) g(Y, \phi W) \\
& -g(X, \phi W) g(Y, \phi Z)\} .
\end{aligned}
$$

Moreover, if such a manifold has constant $\phi$-sectional curvature equal to $c$, then its curvature tensor is given by 


$$
\begin{aligned}
R(X, Y) Z=\left(\frac{c+}{4}\right. & \{\alpha \\
& +\left(\frac{c-\alpha}{4}\right)\{g(X, \phi Y) \phi Z-g(Y, \phi Z) \phi X \\
& +2 g(X, \phi Y) \phi Z-\eta(X \eta(Z) Y \\
& +\eta(Y) \eta(Z) X-g(X, Z) \eta(Y) \xi \\
& -g(Y, Z) \eta(X) \xi\}
\end{aligned}
$$

for any $\boldsymbol{X}, \boldsymbol{Y}, \boldsymbol{Z} \in \boldsymbol{\chi}(\boldsymbol{M})$.

A normal almost $C(\alpha)$ - manifold is said to be a $C(\alpha)$-manifold. For example, Co-Kählerian, Sasakian and Kenmotsu manifolds are $C(0), C(1)$ and $C(-1)$-manifolds, respectively [3].

\section{Theorem 2.1.}

(i) An almost co-Hermitian manifold $\boldsymbol{M}$ is $\boldsymbol{\alpha}$-Sasakian if and only if

$$
\left(\nabla_{X} \phi\right) Y=\alpha\{g(X, Y) \xi-\eta(X) Y\},
$$

for all $X, Y \in \chi(M)$.

(ii) If $M$ is $\alpha$-Sasakian, then $\xi$ is Killing vector field and

$$
\nabla_{X} \xi=-\alpha \phi X
$$

(iii) An $\boldsymbol{\alpha}$-Sasakian manifold is a $\boldsymbol{C}\left(\boldsymbol{\alpha}^{2}\right)-$ manifold [3].

\section{Theorem 2.2.}

(i) An almost co-Hermitian manifold $\boldsymbol{M}$ is an $\boldsymbol{\alpha}$-Kenmotsu manifold if and only if

$$
\left(\nabla_{X} \phi\right) Y=\alpha\{g(\phi X, Y) \xi-\eta(Y) \phi X\},
$$

for all $X, Y \in \chi(M)$.

(iii) An $\boldsymbol{\alpha}-$ Kenmotsu manifold is a $\boldsymbol{C}\left(-\boldsymbol{\alpha}^{2}\right)-$ manifold.

\section{An Almost $C(\alpha)$-Manifold Satisfying Certain Conditions on the Concircular Curvature Tensor}

In this section, we will give the main results for this paper.

Let $\boldsymbol{M}$ be a $(\mathbf{2 n}+\mathbf{1})-$ dimensional almost $\boldsymbol{C}(\boldsymbol{\alpha})$-manifold and denote Riemannian curvature tensor of $\boldsymbol{R}$, then we have from (2.2), for $\mathbf{X}=\boldsymbol{\xi}$

$$
\boldsymbol{R}(\xi, Y) Z=\alpha\{g(Y, Z) \xi-\eta(Z) Y\} .
$$

In the same way, choosing $\mathbf{Z}=\xi$ in (2.2), we have

$$
\boldsymbol{R}(\boldsymbol{X}, \boldsymbol{Y}) \boldsymbol{\xi}=\boldsymbol{\alpha}\{\boldsymbol{\eta}(\boldsymbol{Y}) \boldsymbol{X}-\boldsymbol{\eta}(\boldsymbol{X}) \boldsymbol{Y}\} .
$$

In (3.2), choosing $\mathbf{Y}=\boldsymbol{\xi}$, we obtain

$$
\boldsymbol{R}(X, \xi) \xi=\alpha\{X-\eta(X) \xi\} .
$$

$$
\boldsymbol{\eta}(\boldsymbol{R}(\boldsymbol{X}, \boldsymbol{Y}) \boldsymbol{Z})=\boldsymbol{\alpha}\{\boldsymbol{g}(\boldsymbol{Y}, \boldsymbol{Z}) \boldsymbol{\eta}(\boldsymbol{X})-\boldsymbol{g}(\boldsymbol{X}, \boldsymbol{Z}) \boldsymbol{\eta}(\boldsymbol{Y})\}
$$

From (2.2), we can state

$$
\begin{aligned}
R\left(X, e_{i}\right) e_{i}+R(X, & \left.\phi e_{i}\right) e_{i}+R(X, \xi) \xi \\
& =\left(\frac{c+3 \alpha}{4}\right)\left\{n X-g\left(X, e_{i}\right) e_{i}+n X\right. \\
& \left.-g\left(X, \phi e_{i}\right) \phi e_{i}+X-g(X, \xi) \xi\right\} \\
& +\left(\frac{c-\alpha}{4}\right)\left\{3 g\left(X, \phi e_{i}\right) \phi e_{i}-2 n \eta(X) \xi\right. \\
& +3 g\left(X, \phi^{2} e_{i}\right) \phi^{2} e_{i} \eta(X) \xi \\
& -X\} \quad(3.5)
\end{aligned}
$$

for $\left\{e_{1}, e_{2}, \ldots, \phi e_{1}, \phi e_{2}, \ldots, \phi e_{n}, \xi\right\}$ orthonormal basis of $\boldsymbol{M}$. From (3.5), for $\in \boldsymbol{\chi}(\boldsymbol{M})$, we obtain

$S(X, Y)$

$$
\begin{aligned}
& =\left(\frac{\alpha(3 n-1)+c(n+1)}{2}\right) \\
& +\left(\frac{(\alpha-c)(n+1)}{2}\right) \eta(X) \eta(Y)
\end{aligned}
$$

which is equivalent to

$Q X$

$$
\begin{aligned}
& =\left(\frac{\alpha(3 n-1)+c(n+1)}{2}\right) X \\
& +\left(\frac{(\alpha-c)(n+1)}{2}\right) \eta(X) \xi
\end{aligned}
$$

From (3.7) we can give the following corollary.

Corollary 3.1. An almost $\boldsymbol{C}(\boldsymbol{\alpha})$-manifold is always an $\boldsymbol{\eta}$-Einstein manifold.

Also, from (3.6), we can easily see

$$
\begin{array}{r}
\tau=n[\alpha(3 n+1)+c(n+1)], \\
S(X, \xi)=2 n \alpha \eta(X)
\end{array}
$$

and

$$
Q \xi=2 n \alpha \xi .
$$

Definition 3.1. Let $(\boldsymbol{M}, \boldsymbol{g})$ be an $(\mathbf{2 n}+\mathbf{1})$-dimensional Riemannian manifold. Then the Weyl concircular curvature tensor $\widetilde{\boldsymbol{Z}}$ is defined by

$$
\begin{aligned}
& \widetilde{Z}(X, Y) W=R(X, Y) W \\
&-\frac{\tau}{2 n(2 n+1)}\{g(Y, W) X \\
&-g(X, W) Y\}
\end{aligned}
$$

for all $X, Y, W \in \chi(M)$, where $\tau$ is the scalar curvature of $M[5]$.

In (3.11), choosing $X=\xi$, we obtain

Also from (3.2), we obtain 


$$
\tilde{Z}(\xi, Y) W=\left(\begin{array}{c}
\left.\alpha-\frac{\tau}{2 n(2 n+1)}\right)\{g(Y, W) \xi \\
-\eta(W) Y\}
\end{array}\right.
$$

Theorem 3.1. Let $M$ be a $(2 n+1)$-dimensional an almost $C(\alpha)$-manifold. Then $\tilde{Z}(\xi, X) R=0$ if and only if $M$ either has $c$ - sectional curvature or the scalar curvature $\tau=$ $2 n \alpha(2 n+1)$.

Proof: Suppose that $\tilde{Z}(\xi, X) R=0$. Then from (3.11), we have

$$
\begin{aligned}
\widetilde{(Z}(\xi, X) R)(U, W) Z & =\tilde{Z}(\xi, \mathrm{X}) \mathrm{R}(\mathrm{U}, \mathrm{W}) \mathrm{Z}-\mathrm{R}(\tilde{Z}(\xi, \mathrm{X}) \mathrm{U}, \mathrm{W}) \mathrm{Z} \\
& -R(U, \tilde{Z}(\xi, X) W) R-R(U, W) \tilde{Z}(\xi, X) Z \\
& =0 .
\end{aligned}
$$

Using (3.12) in (3.13), we obtain

$$
\begin{aligned}
=\left(\alpha-\frac{\tau}{2 n(2 n+1)}\right) & \{g(X, R(U, W) Z) \xi-\eta(R(U, W) Z) X \\
& -g(X, U) R(\xi, W) Z+\eta(U) R(X, W) Z \\
& -g(X, W) R(U, \xi) Z+\eta(W) R(U, X) Z \\
& -g(X, Z) R(U, W) \xi+\eta(Z) R(U, W) X\} \\
& =0 .
\end{aligned}
$$

Using (3.2), (3.3) and putting $U=\xi$ in (3.14), we get $\left(\alpha-\frac{\tau}{2 n(2 n+1)}\right)\{R(X, U) W-\alpha\{g(U, W) X-g(X, W) U\}\}=0$. Therefore, manifold has either $\alpha$-sectional curvature or $\tau=2 n \alpha(2 n+1)$. This implies that $\alpha=c$.

Theorem 3.2. Let $M$ be a $(2 n+1)$-dimensional an almost $C(\alpha)$-manifold. $\tilde{Z}(\xi, X) \tilde{Z}=0$ if and only if $M$ either has $\alpha$ - sectional curvature or the scalar curvature $\tau=$ $2 n \alpha(2 n+1)$.

Proof: Suppose that $\tilde{Z}(\xi, X) \tilde{Z}=0$, we have

$\widetilde{(Z}(\xi, X) \tilde{Z})(Y, U) W=\tilde{Z}(\xi, \mathrm{X}) \tilde{Z}(\mathrm{Y}, \mathrm{U}) \mathrm{W}-\tilde{Z}(\tilde{Z}(\xi, \mathrm{X}) \mathrm{Y}, \mathrm{U}) \mathrm{W}$

$$
-\tilde{Z}(Y, \tilde{Z}(\xi, X) U) W-\tilde{Z}(Y, U) \tilde{Z}(\xi, X) W
$$$$
=0 \text {. }
$$

Using the equations (3.12) and (3.2), (3.3) in (3.15), we have $=\tilde{Z}(\xi, \mathrm{X}) \mathrm{R}(\mathrm{Y}, \mathrm{U}) \mathrm{W}-\tilde{Z}(\mathrm{R}(\xi, \mathrm{X}) \mathrm{Y}, \mathrm{U}) \mathrm{W}$

$$
\begin{aligned}
& -\tilde{Z}(Y, R(\xi, X) U) W-\tilde{Z}(Y, U) R(\xi, X) W \\
& +\left(\alpha-\frac{\tau}{2 n(2 n+1)}\right)\{g(Y, W) \tilde{Z}(\xi, X) U \\
& -g(U, W) \tilde{Z}(\xi, X) Y+g(X, Y) \tilde{Z}(\xi, U) W \\
& -\eta(Y) \tilde{Z}(X, U) W+g(X, U) \tilde{Z}(Y, \xi) W \\
& -\eta(U) \tilde{Z}(Y, X) W+g(X, W) \tilde{Z}(Y, U) \xi \\
& -\eta(W) \tilde{Z}(Y, U) X\}
\end{aligned}
$$

$=0$.

Putting $Y=\xi$ in (3.16), we get

$\left(\alpha-\frac{\tau}{2 n(2 n+1)}\right)\{R(X, U) W-\alpha\{g(U, W) X-g(X, W) U\}\}=0$.

This tell us that $\boldsymbol{M}$ has either $\boldsymbol{\alpha}$-sectional curvature or the scalar curvature $\tau=\mathbf{2 n} \boldsymbol{\alpha}(\mathbf{2 n}+\mathbf{1})$.

The converse is obvious.

Theorem 3.3. Let $\mathbf{M}$ be a $(2 \mathbf{n}+\mathbf{1})$-dimensional an almost $\mathbf{C}(\boldsymbol{\alpha})-$ manifold. Then $\widetilde{\mathbf{Z}}(\boldsymbol{\xi}, \mathbf{X}) \mathbf{S}=\mathbf{0}$ if and only if $\boldsymbol{M}$ reduce an Einstein manifold.

Proof: We suppose that $\widetilde{\mathbf{Z}}(\boldsymbol{\xi}, \mathbf{X}) \mathbf{S}=\mathbf{0}$, which implies that

$$
\boldsymbol{S}(\widetilde{\mathbf{Z}}(\xi, \mathbf{X}) \mathbf{U}, \mathbf{W})=+\boldsymbol{S}(\boldsymbol{U}, \widetilde{\mathbf{Z}}(\xi, X) W)=\mathbf{0} .
$$

$$
\begin{aligned}
& \boldsymbol{S}\left(\boldsymbol{R}(\xi, X) U-\frac{\tau}{2 n(2 n+1)}\{g(X, U) \xi-\eta(U) X\}, W\right) \\
+ & \boldsymbol{S}\left(\boldsymbol{U}, \boldsymbol{R}(\xi, X) W-\frac{\tau}{2 n(2 n+1)}\{g(X, W) \xi-\eta(W) X\}\right) \\
= & 0
\end{aligned}
$$

Using (3.1), (3.9) in (3.18), we obtain

$$
=2 n \alpha^{2} g(X, U) \eta(W)-\alpha \eta(U) S(X, W)
$$$$
-\frac{\tau}{2 n(2 n+1)}(2 \operatorname{nag}(X, U) \eta(W)-\eta(U) S(X, W))
$$$$
+2 n \alpha^{2} g(X, W) \eta(U)-\alpha \eta(W) S(X, U)
$$

$$
\begin{aligned}
& -\frac{\tau}{2 n(2 n+1)}(2 n \alpha g(X, W) \eta(U)-\eta(W) S(X, U)) \\
& =0 .
\end{aligned}
$$

Putting $\boldsymbol{U}=\boldsymbol{\xi}$ in (3.19), we get

$$
\begin{aligned}
& S(X, W)\left\{\frac{\tau}{2 n(2 n+1)}-\alpha\right\}+g(X, W)\left\{2 n \alpha^{2}-\alpha \frac{\tau}{2 n+1}\right\} \\
& =0
\end{aligned}
$$

$$
\begin{aligned}
\text { under the condition } \alpha \neq \frac{\tau}{2 n(2 n+1)}, \\
S(X, W)=2 n \alpha g(X, W) .
\end{aligned}
$$

Therefore, the manifold is Einstein manifold.

The converse is obvious.

If $\boldsymbol{M}$ is an Einstein manifold, the scalar curvature $\boldsymbol{\tau}$ of $\boldsymbol{M}$ is

$$
\tau=2 n \alpha(2 n+1) \text {. }
$$

By corresponding (3.8) and (3.20) we obtain $\boldsymbol{\alpha}=\boldsymbol{c}$ which implies that $\boldsymbol{M}$ is of constant sectional curvature $\boldsymbol{c}$.

Definition 3.2. Let $(\mathbf{M}, \mathbf{g})$ be a $(\mathbf{2} \mathbf{n}+\mathbf{1})-$ dimensional Riemannian manifold. Then Weyl projective curvature tensor $\boldsymbol{P}$ is defined by

$$
\begin{aligned}
P(X, Y) Z & =R(X, Y) Z \\
& -\frac{1}{2 n}\{S(Y, Z) X-S(X, Z) Y\},
\end{aligned}
$$

where $\boldsymbol{R}$ is Riemannian curvature tensor and $\boldsymbol{S}$ is Ricci tensor [5].

Theorem 3.2. Let $\boldsymbol{M}$ be a $(2 n+1)$-dimensional an almost $\boldsymbol{C}(\boldsymbol{\alpha})$ - manifold. Then, $\widetilde{\mathbf{Z}}(\boldsymbol{\xi}, \mathbf{X}) \mathbf{P}=\mathbf{0}$ if and only if $\boldsymbol{M}$ reduce an Einstein manifold.

Proof: Suppose that $\widetilde{\mathbf{Z}}(\boldsymbol{\xi}, \mathbf{X}) \mathbf{P}=\mathbf{0}$. Then we have, $\widetilde{(Z}(\xi, X) \mathrm{P})(Y, U) W=\tilde{Z}(\xi, \mathrm{X}) \mathrm{P}(\mathrm{Y}, \mathrm{U}) \mathrm{W}-\mathrm{P}(\tilde{Z}(\xi, \mathrm{X}) \mathrm{Y}, \mathrm{U}) \mathrm{W}$

$$
\begin{aligned}
& -\mathrm{P}(Y, \tilde{Z}(\xi, X) U) W-\mathrm{P}(Y, U) \tilde{Z}(\xi, X) W \\
& =0,
\end{aligned}
$$

for $X, Y, U, W \in \chi(M)$. Using (3.12) in (3.22), we get

$$
\left(\alpha-\frac{\tau}{2 n(2 n+1)}\right)\{\alpha g(X, U) \eta(W) \xi-\alpha g(U, W) X+
$$

Using (3.12) in (3.17), we get 


$$
\begin{aligned}
& \frac{1}{2 n}\{S(U, W) X+S(X, W) \eta(U) \xi-S(U, X) \eta(W) \xi\} \\
& -\alpha g(X, W) \eta(U) \xi+P(U, X) W\}
\end{aligned}
$$

$=\mathbf{0}$.

Taking inner product both sides of (3.23) by $\xi \in \boldsymbol{\chi}(\boldsymbol{M})$, we obtain

$$
\begin{aligned}
& \left(\alpha-\frac{\tau}{2 n(2 n+1)}\right)\{\alpha g(X, U) \eta(W)-\alpha g(U, W) \eta(X)+ \\
& \frac{\mathbf{1}}{\mathbf{2 n}}\{\boldsymbol{S}(\boldsymbol{U}, \boldsymbol{W}) \boldsymbol{\eta}(\boldsymbol{X})+\boldsymbol{S}(\boldsymbol{X}, \boldsymbol{W}) \boldsymbol{\eta}(\boldsymbol{U})-\boldsymbol{S}(\boldsymbol{U}, \boldsymbol{X}) \boldsymbol{\eta}(\boldsymbol{W})\} \\
& -\boldsymbol{\alpha} \boldsymbol{g}(\boldsymbol{X}, \boldsymbol{W}) \boldsymbol{\eta}(\boldsymbol{U})+\boldsymbol{\eta}(\boldsymbol{P}(\boldsymbol{U}, \boldsymbol{X}) \boldsymbol{W})\}
\end{aligned}
$$

$\mathbf{0} \mathbf{0}$.

Also making use of (3.21), we obtain

$$
\begin{array}{r}
\boldsymbol{\eta}(\boldsymbol{P}(\boldsymbol{U}, \boldsymbol{X}) \boldsymbol{W})=\boldsymbol{\alpha}\{\boldsymbol{g}(\boldsymbol{X}, \boldsymbol{W}) \boldsymbol{\eta}(\boldsymbol{U})-\boldsymbol{g}(\boldsymbol{U}, \boldsymbol{W}) \boldsymbol{\eta}(X)\} \\
-\frac{1}{2 n}\{\boldsymbol{S}(X, W) \boldsymbol{\eta}(U)-S(U, W) \boldsymbol{\eta}(X)\}
\end{array}
$$

Using (3.25) in (3.24) and choosing $\boldsymbol{W}=\boldsymbol{\xi}$, we have provided that $\alpha \neq \frac{\tau}{2 n(2 n+1)}$,

$$
S(U, X)=2 n \alpha g(U, X) \text {. }
$$

So, the manifold is an Einstein manifold. The converse is obvious.

\section{References}

[1] C. Özgür and M. M. Tripathi, On P-Sasakian manifolds satisfying certain conditions on the concircular curvature tensor, Turkish Journal of Math. , 31(2007), $171-179$.

[2] D. E. Blair, J. S. Kim and M. M. Tripathi, On concircular curvature tensor of a contact metric manifold, J. Korean Math. Soc. 42(2005), 883-892.

[3] D. Janssens and L. Vanhecke, Almost contact structure and curvature tensors, Kodai Math.J., 4(1981), 1-27.

[4] D. Perrone, Contact Riemannian manifolds satisfying R(X, $\xi) \cdot R=0$, Yokohama Math. J. 39 (1992), 2, 141-149.

[5] K. Yano and M. Kon, Structures on manifolds, Series in Pure Math., Vol. 3, Word Sci., (1984).

[6] K. Yano, Concircular geometry I. Concircular transformations, Proc. Imp. Acad. Tokyo 16 (1940), 195-200.

[7] M. M. Tripathi and J. S. Kim, On the concircular curvature tensor of a $(\kappa, \mu)$-manifold, Balkan J. Geom. Appl. 9, no.1, 104 - 114 (2004).

[8] Z. I. Szabo, Structure theorems on Riemannian spaces satisfying $\mathrm{R}(\mathrm{X}, \mathrm{Y}) \cdot \mathrm{R}=0$, the local version, Diff. Geom., 17(1982), 531-582. 